\title{
MEDICAL NUTRITION THERAPY
}

\author{
Bhattarai Jyoti \\ TUTH, Visiting Faculty, Department of Internal Medicine \\ Kathmandu, Nepal
}

\section{INTRODUCTION}

Diabetes can lead to devastating expensive complications such as eye blindness, heart disease, stroke, leg amputation etc. To prevent such complications of diabetes it is really important that health care workers including patients understand the ways of improving or reducing the chances of developing such complications. It has been proven that proper management of diabetes early on can reduce such expensive and painful complications ${ }^{1}$.

Proper management comprises of lifestyle modifications that include proper exercise, balanced nutritious diet, taking pills on regular basis, getting regular check-ups and keeping different parameters of the health in recommended range.

Lifestyle modification is an integral part of diabetes management. It comprises of regular exercise and proper nutrition. Proper nutrition is very important in diabetes care. When nutrition education is given and applied on a day to day, basis, with the intention of improving disease care and improving longevity and quality of life, it is called medical nutrition therapy.

\section{Nutrition}

Nutrition is an assortment of different quantity and quality of nourishing elements that are found in food. People eat food to obtain nourishing essential elements for sustaining life, to satisfy their taste buds and also for social reasons. If people are not empowered by appropriate knowledge they may end up eating wrong quality and quantity of food that may be negatively impacting their diabetic care. Medical nutrition therapy helps the patients get empowered and learn to make the right choices. When they chose the right quality and quantity of food their sugar and cholesterol level can be maintained in desired range. They can go on about their lives with happiness and joy and at the same time help prevent devastating complications of diabetes.

\section{Types of nutrients obtained from human diet} There are various kinds of essential elements that we get from food. We totally depend on our food for energy source but if the energy source is too much or too little it can negatively impact our health. The macronutrients called fat, protein and carbohydrate are the main sources of energy from human diet. We also get vitamins and minerals that are essential for different reactions occurring in the body. Before understanding medical nutrition therapy and their roles in diabetes we need to understand what type of nutrients we get from the food we eat.

We mainly get two types of nutrients from our food; macronutrients and micronutrients:

\section{Macronutrients}

Macronutrients are the main backbone of our nutrition and they provide the bulk of our energy. Macro nutrients are carbohydrate, protein and fat. They are the main sources of energy from human diet.

\section{Micronutrients}

Micronutrients are nutrients that are necessary in minute quantities. Still they are essential for our overall health. Examples are vitamins and minerals.

\section{Fiber and Water}

In addition to macronutrients and micronutrients we get essential elements from our food such as fiber and water. Our body is made up of two third water and water is essential for our existence. 


\section{Units of energy:}

Unit of energy is expressed in kilo calories or in kilo joules. Energy density of food or diet is metabolizable energy per unit per weight of food ${ }^{2}$. Fat is a high energy density food because onegram fat gives nine kilo calories whereas one gram of protein or carbohydrate source gives four kilo calories. There are other fruits and vegetable which may be having low energy density. For example, cucumber has a lot of water in it. Cucumber has minimal amount of carbohydrate and protein and no fat at all. Thus it is a low density food. It is important to understand the energy density of food so that we can incorporate this information in the medical nutrition therapy.

\section{Carbohydrate}

Carbohydrate is the most common source of stable food in many parts of the world. Carbohydrate is generally obtained from cereals and to a lesser degree from pulses and fruits. The basic unit of carbohydrate is a simple sugar. Examples of simple sugars are fructose, galacatose and glucose. If one simple sugar is combined with another simple sugar it is called disaccharide such as sucrose and lactose. When there is a big polymer of multitude of simple sugar it is called polysaccharides. There are two types of polysaccharides i.e. starch and non-starch polysaccharides. Nonstarch polysaccharides are generally soluble plant carbohydrates and they are mostly considered fiber. They pass through the gut without absorption and they are considered important to form the bulk of the stool and also cause slow absorption of other carbohydrates.

\section{Protein}

Protein is one important nutrient that we get from our food. It is nitrogen containing component of diet. It is made up of various amino acids. Protein is obtained from both animal and plant source. It is broken down into amino acid by gut enzymes and absorbed in the system. It is essential to synthesize various compounds in the body. We need continuous replacement of protein for wear and tear so our food should have certain amount of protein in the diet.

\section{Fat}

Fat is an essential nutrient in our food. We get fat from both animal and plant sources. But fat from plant source is considered better, except for few exceptions such as palm oil, coconut oil and hydrogenated vegetable oils. Fat is also called lipid and it is insoluble in water. Fat or lipid is essential for the integrity of cell wall and essential for transportation of vitamins such as A, D, E and K. The smallest unit of fats or lipids is called fatty acid. There are various types of fatty acids obtained from food i.e. saturated fatty acids, polyunsaturated fatty acids and mono-un-saturated fatty acids. Saturated fatty are those fatty acids which have all the available bonds of carbon occupied. Mono unsaturated fatty acids have a single point of unsaturation. Example of mono saturated fatty acid is oleic acid. Poly unsaturated fatty acids have many double bonds in carbon chain the example of which is ecosapenta ionic acids. Saturated fatty acids are considered to be more harmful than mono-un-saturated fatty acid and poly unsaturated fatty acids.

\section{Trans fatty acids}

When unsaturated fatty acid is converted to saturated fatty acid with the help of reaction with hydrogen the process is called hydrogenation. Fatty acids, which are hydrogenated, are called Trans fatty acids. Trans fatty acids are harmful to the body.

Animal fat have more saturated fatty acids. Plant based oils such as soya oil, mustard oil, olive and sunflower oil have high amount of poly unsaturated fatty acids. Fish oil is also a source of unsaturated fatty acid and they have ecosapenta ionic acid and DHA. Those fatty acids which have unsaturated bonds are considered to be healthy for human diet. As fat is high in energy, altering the quality and quantity of fat can improve your energy intake. Fat is a high energy density of food, as it can give a large amount of energy, the recommendation is to eat low fat diet .

\section{Definition of Medical nutrition therapy}

Medical Nutrition Therapy is defined as nutritional, 
diagnostic, therapeutic and counseling services for the purpose of disease management which is provided by registered dietician or nutritional professional or physician3. Medical nutritional therapy is a legal term that is mainly used in the United States. Nutritional therapy' is the term that is used in various studies that have been done outside of the US. Thus it will be more inclusive of the broad spectrum of recommendations that fall under dietary education and management offered to patients to manage diabetes. Nutrition therapy is very important for patients with diabetes and pre-diabetes. Dietary intervention in diabetes has proven that the hemoglobin A $1 \mathrm{C}$ can be reduced with the help of nutrition therapy ${ }^{4}$.

Nutrition therapy provided by a registered dietician has been found to be associated with decrease of hemoglobin A1C of $0.3-1 \%$ of people with type 1 diabetes (4) and $0.5 \%-2 \%$ for people with type 2 diabetes5.

\section{Important components of Medical Nutrition Therapy or Nutrition Therapy are6}

1. Nutrition Assessment

2. Nutrition Diagnosis

3. Nutrition Interventions

4. Nutrition Monitoring and Evaluation with ongoing follow up to support long term lifestyle changes

5. Evaluation of Outcomes and Modify interventions as needed.

Providing Nutrition Therapy to individuals is challenging in resource poor countries. Even in more developed countries it is an under unitized tool of diabetes management. One study showed that only $9 \%$ people interviewed and one diet visit in the last one year ${ }^{7}$.

\section{The goals of nutrition therapy8}

The goal of nutrition therapy is to:

1.To promote and support healthful eating pattern

2. Maintain healthy weight and various parameters of diabetes care in the desired range such as fasting, pp (post prandial) blood sugar, hemoglobin A1C, cholesterol,blood pressure and waist circumference 3. Maintain pleasure of eating by providing positive messages about food choices

4.Delay or prevent the devastating complications of diabetes.

5. Provide individuals with practical tools for day to day meal planning rather than focusing in individual micro-nutrient or single food.

6. Uplift the feeling of wellbeing and carry the daily activities.

7. Avoiding unhealthy intake of harmful substances like smoking and alcohol

\section{Macro nutrient percentage:}

People have widely differing energy intake and proportional energy contribution from carbohydrate, protein and fat sources.

One study showed that in the US, people with type 1 and type 2 diabetes report and obtaining about $45 \%$ of total energy from carbohydrate, $35-40 \%$ from fat and 16-18\% energy intake from protein ${ }^{9}$.

There is no ideal percentage of total energy derived from one particular type of macronutrient. There is no evidence to suggest clearly that, sticking to strict percentage of calories from carbohydrate, protein and fat for all person of diabetic is helpful ${ }^{8}$. Macronutrient distribution should be based on individualized assessment of current eating patterns, preferences and metabolic goals ${ }^{8}$.

\section{Energy Intake (Energy Balance)}

Various studies have shown that intensive lifestyle modification in patients with impaired glucose tolerance or diabetes can lead to weight loss, which in turn leads to better sugar control and better lipid panel 10,11,12. Weight loss has been shown to reduce hemoglobin $\mathrm{A} 1 \mathrm{C}$ and blood sugar profile. In the Look AHEAD trial, intensive lifestyle intervention was associated with weight loss of $6 \%$ at the end of intervention. The study population had to be seen 3-4 times monthly over a year and 1-2 times each month for the reminder of the trial. The total trial was of the period of 9.6 years 13 . Look AHEAD calorie goals were 1200-1500 Kcal/ day for weights less than $114 \mathrm{~kg}$ group and 1500 - 
$1800 \mathrm{kcal} /$ day for more than $114 \mathrm{~kg}$ group and they were provided with less than $30 \%$ fat ${ }^{14}$. In resource poor developing countries, with lack of health professionals and dietitians, this type of intensive consultation will definitely be challenging and out of reach for many people.

\section{Weight loss diet and outcome in Diabetes Mellitus type 2}

In type 2 patient who has obesity or central obesity, modest weight loss defined as weight loss of more than $5 \%$ is needed to produce desired outcome in glycemic control. Weight loss can be obtained with lifestyle program that leads to $500-750 \mathrm{~kg} /$ calories negative energy balance or provide about 1200 $-1500 \mathrm{kcal} /$ day for women and 1500-1800 kcal for men $^{15}$.

\section{Carbohydrate}

As carbohydrate containing food are the ones contributing to the post meal blood sugar level, it is very important to know which food contains carbohydrate.

\section{Carbohydrate counting and diabetes management}

For individuals who take secretagogues or insulin, to reduced risk of hypoglycemia, it is recommended to eat source of carbohydrate at every meal. Skipping meal is not recommended. As physical activity can lower the blood sugar abruptly, it is recommended for them to carry carbohydrate with them all the time to reduce the risk of hypoglycemia.

For individuals with type-1 diabetes, it is recommended to count carbohydrate and match it at the meal time insulin. Meals need to be consumed at similar times every day and insulin needs to be taken before the meal. It is recommended to eat similar amount of carbohydrate everyday to match the set dose of insulin.

When type 1 patients are taught about carbohydrate counting and matching the carbohydrate intake with short acting insulin, their $\mathrm{HbAlc}$ has been found to be lower16. In places with poor socioeconomic condition, lower education level and understanding this type of education may be difficult. Alternative simpler way of teaching them portion sizes has been found to be effective. It may be better especially for elderly individuals and those with cognitive dysfunction.

Example of simple ways of educating people about diabetes nutrition amount and portion size may be plate method or Zimbabwean hand jive model as proposed by Dr.K Mawji. In Zimbabwean hand jive model, starches or carbohydrate containing food to be taken in one meal is the amount that can hold in 2 fists. The amount covering the palm of one hand is for protein containing foods. And green nonstarch vegetables, will be as much as can be held by 2 hands. Oil is to be taken less than equivalent to tip of the thumb.

In plate method, non-starchy vegetables fill up the half of a 9 inches' plate. Of the remaining half, one quarter of the plate has to be filled by grains and starchy food, which are actually the main source of carbohydrate. The remaining quarter is for protein containing foods such as meat and dairy. One can add healthy fat such as vegetable oil, nuts in small amount. Add a serving of fruit and a non caloric drink such as water, tea or coffee.

\section{Glycemic index}

According to ADA review, the association of glycemic index and glycemic loads with diabetic is complex (7). The concept of glycemic index improving the diabetic management has not been substantiated. A meta-analysis showed that some studies exhibit that intake of lower glycemic intake carbohydrate can cause reduction of hemoglobin A $1 \mathrm{C}$ of $-0.2 \%$ to $-0.5 \%$, though in these studies, there was no consistent definition of low glycemic index food ${ }^{17}$.

\section{Dietary fiber and whole grain}

A systemic review of data did not show a significant improvement of glycemic control with high fiber diet18. High dietary fiber showed a modest lowering 
of pre-prandial glucose and hemoglobin A1c $(-0.2$ to- $-0.3 \%)$ with more than $50 \mathrm{gm}$ fiber /day19. ADA position statement on nutrition suggests that based on existing research, acquiring fiber intake of $50 \mathrm{gm} /$ day is unrealistic. Dietary fiber is associated with lower all cause mortality, thus people with diabetes are advised to consume at least the amount of fiber and whole grains recommended for general public, i.e. 14 gm fiber per 1000kal per day ${ }^{8}$.

\section{Fructose}

Fructose is obtained from fruit is called free fructose'. Free fructose is naturally occurring and obtained from fruits. As long as its intake is not excessive, it is not detrimental. But fructose present in corn syrup that are added in factory made food such as sugary drinks have, should not be consumed to avoid excessive calories intake. High fructose syrup containing diet has been associated with dyslipidemia ${ }^{20}$.

\section{Protein intake}

Research has not shown clearly the ideal amount of protein intake for optimized glycemic control 15. Protein improves satiety and should be part of the diet. However intake goal should be individualized. $15-20 \%$ of total energy intake from protein is recommended to patients with diabetes as it is to general population ${ }^{15}$.

People who already have diabetic kidney disease are not recommended complete restriction of protein. Protein intake should be maintained at 0.8 gram/ kg per body weight per day. Protein restriction below $0.8 \mathrm{gram} / \mathrm{kg}$ per body weight per day is not recommended ${ }^{15}$.

Protein enhances insulin secretion in type 2 diabetes patients, high protein containing carbohydrate source food should not be used to treat hypoglycemia ${ }^{15}$.

\section{Fat intake and Diabetes}

Diabetes organizations in various countries recommend optimal diet for patients with diabetes that has less than $30 \%$ of energy intake from fat with moderate polyunsaturated fat and restriction of saturated and unsaturated fat ${ }^{21}$.

The supplementation of omega-3 fatty acid is not recommended whereas obtaining polyunsaturated fatty acids and monounsaturated fatty acids instead of saturated fatty acids and trans-fatty acids from food sources is recommended8. Systemic review about dietary supplement with omega 3 fatty acids did not show improvement of glycemic control in type 2 diabetic $^{17}$.

\section{Various dietary approaches in diabetes}

People in different countries and various sociocultural situations have different food combinations and eating patterns. When people eat combinations of food, they will be getting different combinations of micro and macro nutrients. Various studies have been done with the hope of finding best diet combination for patients with diabetes.

\section{Different types of diet combinations studied in Diabetes}

Mediterranean diet includes plant foods, minimally processed, locally grown food, fresh fruits, limited concentrated sugar, olive oil as main source of dietary lipid. It has low to moderate amount of dairy products such as cheese and yogurt, less than four eggs per week, red meat in low frequency and amount. According to one meta-analysis, Mediterranean diet compared to other diet improved glycemic control by reducing hemoglobin A1c of about $-0.47 \%{ }^{21}$.

Low fat diet consists of vegetables, fruit and starches. Protein and low fat dairy products ${ }^{22}$. Total fat intake is less than $30 \%$ of total energy intake and saturated fat intake is less than $10 \%{ }^{23}$. Low fat diet can be beneficial when overall energy intake is reduced and the weight loss happens.

Low carbohydrate diet has high protein intake such as meat, fish, chicken, egg, cheese, nuts and seeds, fats from oil, butter, olive and vegetables low in carbohydrate such as greens. Sugar containing food and grain products such as pasta, rice and bread are avoided. In research studies, the definition of 
very low carbohydrate diet is the one that has 21 70 gram per day of carbohydrate and moderately low carbohydrate one that has $30-40 \%$ of calories contribution from carbohydrate ${ }^{24}$.

A meta-analysis of low carbohydrate diet compared with other diet showed a decrease in hemoglobin A1c of about $-0.12 \%{ }^{21}$.

High protein diet has 30\% energy intake from protein, $40 \%$ from carbohydrate, $30 \%$ from fat with 20 gm protein after 2 months. A high protein, low carbohydrate, diet showed about $-0.28 \%{ }^{25}$.

Mediterranean diet has been shown to improve cardiovascular risk factors such as lipid pattern, blood pressure in patients with diabetes. Vegetarian and low fat vegetarian diet did not consistently improve glycemic control or cardiovascular disease. There is no ideal eating pattern that can benefit all diabetes patients. There is no one size fits for all. Thus medical nutrition therapy has to be individualized.

In DASH (The Dietary Approaches to Stop Hypertension) diet you eat fruits, vegetables and low fat dairy products and reduced in sodium level. ${ }^{26}$.

\section{Micronutrients}

Micronutrients are essential for us. They are best obtained from natural sources rather than from supplements. Supplementation with anti oxidants, vitamin $C$, etc is non advised due to lack of evidence of efficacy. Supplementation of micronutrients such as chromium, magnesium and vitamin $\mathrm{D}$, show conflicting and confounding results and thus they are not recommended ${ }^{15}$.

Rather than taking processed food, we recommend high fiber nutrient dense carbohydrate containing food, which provide vitamin and minerals and fiber and lower calories.

Consumption of sugar sweetened beverages, processed foods that are marketed as low fat or non fat food product but that have a high amount of fine grain added sugar should be strongly discouraged ${ }^{15}$.

\section{Sodium}

The recommended daily intake of sodium consumption is less than 2300 milligram per day. Low sodium intake less than 1500 milligram per day is not recommended as it may affect the palatability and it is challenging to achieve that in a nutritionally adequate diet ${ }^{15}$.

\section{Conclusion}

Individualized meal planning to obtain proper percentage of macronutrients is important in nutrition therapy. The food pattern or eating pattern should be chosen according to the patient's taste and cultural habits and palatability. It should be tailored to the patients' need with focus on proper energy balance and also matching with the medication therapy that the patient is taking. Patients should be encouraged to take their meals in scheduled time. They are advised not to skip meals as it can lead to hypoglycemia especially while taking insulin or insulin secreting medication.

Comprehensive nutrition therapy should be an integral part of management of diabetes in all the patients. Due to lack of resources and lack of awareness amongst patients, doctors, policy makers and society as a whole, providing such therapy to all the patients with diabetes may be challenging. It is recommended that the policy makers realize the importance of nutrition therapy and put resources in training and developing registered dieticians, so that as a part of lifestyle modification therapy, it can be offered to the patient. This approach can in turn reduce the burden of diabetes related complications.

\section{Reference for Nutrition Therapy}

1. UKPDS Prospective Diabetes Study (UKPDS) Group. Intensive blood-glucose control with sulphonylureas or insulin compared with conventional treatment and risk of complications in patients with type 2 diabetes. Lancet. 1998; 352;837-853

2. Webb PG . Nutrition maintain and improving health 2012

3. US department of Health and Human Services: Final MNT regulations. CMS- H69FC Federal 
MEDICAL NUTRITION THERAPY

Jour of Diab and Endo Assoc of Nepal 2018; 2 (1): (36-43)

Register 1 November 2001, 42 CFR parts 405, 410, 411, 414 and 415

4. Kulkarni K, Castle G, Gregory R, Holmes A Leontos C, Powers M, Snetselaar L,Splett P, Wylie-Rosett J Nutrition Practice guideline for type 1 diabetes mellitus positively affect dietician practices and patient outcome

5. UKPDS Study Group Response of fasting plasma glucose to diet therapy in newly presenting type II diabetic patients Metabolism 39; 905-912, 1990

6. Lacey K, Pritchett E. Nutrition care process and model: ADA adopts road map to quality care and outcome management.

7. Robbins JM, Thatcher GF. Webb DA, Valdmanis VG. Nutritionist visits, diabetes classes, and hospitalization rates and charges: The Urban Diabetes Study. Diabetes Care 2008; 31:655-660

8. EvertAetal Nutrition Therapy Recommendations for Management of Adults with diabetes : Diabetes Care Volume 36 November 2013

9. Oza-Frank R, Cheng YJ, Narayan KM, Gregg EW. Trends in nutrition intake among adults with diabetes in United States: 1988-2004. J AM Diet Assoc. 2009; 109: 1173-1178

10. Knowler WC, Barrett-Connor E, Fowler SE, et al, Diabetes Prevention Program Research Group. Reduction in the incidence of type 2 diabetes with lifestyle intervention or metformin $\mathrm{N}$ Engl J Med 2002; 346:396-403

11. Tuomilehto J, Lindström J, Eriksson JG, et al, Finnish Diabetes Prevention Study Group. Prevention of type 2 diabetes mellitus by changes in lifestyle among subjects with impaired glucose tolerance. metformin N Engl J Med 2002; 346:396403

12. Look AHEAD research group. Long term effects of lifestyle intervention on weight and cardiovascular risk factors in individuals with type 2 diabetes: four-year results of the look AHEAD trial . Arch Intern Med. 2010; 170; 1566-1575.

13. Look AHEAD research group. Cardiovascular effects of intensive lifestyle intervention in type 2 diabetes. $\mathrm{N}$ Engl J Med. 2013: 369 ; $145-154$

14. Look AHEAD study group: A description of the lifestyle intervention and the evidence supporting it. Obesity 2006; 14(5);737-752

15. Standards of Medical Care in Diabetes -2017; Diabetes Care 2017 Jan; 40

16. DAFNE study group. Training in flexible, intensive insulin management to enable dietary freedom in people with diabetes: dose adjustment for normal eating (DAFNE) randomized controlled trial BMJ 2002;325:746-9

17. Wheeler ML, Dunbar SA, Jaacks LM et al. Macronutrients, food groups and eating patterns in the management of diabetes : a systemic review of the literature 2010 . Diabetes care 2012 ; 35; 434445

18. Franz MJ, Powers MA, Leontos C et al. The evidence for medical nutrition therapy for type 1 and type 2 diabetes in adults. J Am Diet Assoc 2010; 110;1852-1889

19. Jenkins DJ, Kendall CW, Augustin LS et al. Effect of legumes as part of a low glycemic index diet on glycemic control and cardiovascular risk factors in type 2 diabetes mellitus: a randomized controlled trial; Arch Intern Med 2012 Nov 26; 172(21); 1653-60

20. Bantle JP Dietary Fructose and Metabolic Syndrome and Diabetes. The Journal of Nutrition 2009 Jun; 139(6): 1263S -1268S

21. Ajala O, English P, Pinkney J Systemic review and meta-analysis of different dietary approaches to the management of type 2 diabetes; Am J Clin Nutr 2013; 97;505-16

22. Willet WC, Sacks F, Tr ichopoulou A, et al. Mediterranean diet pyramid: a cultural model for healthy eating. Am J Clin Nutr 1995:61:1402S-6S 23. Bernard N, Cohen J, Jenkins A; Low fat diet vegan diet improves glycemic control and cardiovascular risk factors in a randomized clinical trial in individuals with type 2 diabetes. Diabetes Care 2006 Aug; 28(8); 1777-1783

24. Snorgaard O, PoulsenGM, Andersen HK et al. Systemic review and meta-analysis of dietary carbohydrate restriction in patients with type 2 diabetes. BMJ Open Diabetes Research and Care2017;5: e000354 doi;10.1136/bmjdrc-2016

25. Larsen RN, Mann NJ, Maclean E et al. The effect of high-protein, low-carbohydrate diets in 
MEDICAL NUTRITION THERAPY

Jour of Diab and Endo Assoc of Nepal 2018; 2 (1):(36-43)

the treatment of diabetes: a 12 month randomized controlled trial. Diabetologia 2011; 54; 731-40

26. Appel LJ, Thomas JM, Obarzanek W. A Clinical Trial of the Effects of Dietary Patterns on Blood Pressure. N Engl J Med 1997; 336:11171124 